\title{
EFFORT DE PÊCHE ET PRODUCTION EXPLOITÉE DANS LES PETITES RETENUES DU NORD DE LA CÔTE D'IVOIRE.
}

\author{
K.S. DA COSTA (1), K. TRAORE $(1,2)$, L. TITO de MORAIS (3)
}

(1) Institut des Savanes (IDESSA), Station piscicole, B.P. 633, Bouaké 01, Côte d'Ivoire.

(2) Ministère de la Recherche et Technologie, B.P. V 151, Abidjan, Côte d'Ivoire.

(3) Institut français de recherche pour le développement en coopération (ORSTOM). Programme «Petits barrages ", B.P. 1434, Bouaké 01, Côte d'Ivoire.

\section{RÉSUMÉ}

L'effort de pêche et la production piscicole ont été étudiés sur quatre retenues agropastorales du nord de la Côte d'Ivoire : Sambakaha (25 ha) et Gboyo (16 ha) dans la région de Ferké, Kiémou ( 6 ha) et Korokara serpent ( $15 \mathrm{ha}$ ) dans la région de Korhogo. Les études réalisées montrent que la pêche est pratiquée essentiellement par des Bozos originaires du Mali. La densité de pêcheurs par barrage, entre 4 et 17 pêcheurs $\cdot \mathrm{km}^{2}$, est nettement au-dessus de la norme FAO qui est de 2 pêcheurs $\mathrm{km}^{-2}$. L'effort de pêche est très variable d'une retenue à l'autre et la production piscicole est comprise entre 70 et $224 \mathrm{~kg} \cdot \mathrm{ha}^{-1} \cdot \mathrm{an}^{-1}$. Elle est du même ordre de grandeur que celle enregistrée dans des retenues comparables de la région. Les captures restent cependant inférieures aux captures potentielles indiquées par la FAO pour des plans d'eau de ce type (entre 100 et $750 \mathrm{~kg} \cdot \mathrm{ha}^{-1} \cdot \mathrm{an}^{-1}$ ), ce qui montre qu'il peut exister une marge pour augmenter la production piscicole de ces barrages. Les captures estimées sur la totalité des petites retenues représentent entre 16 et $46 \%$ des captures totales de poissons d'eau douce en Côte d'Ivoire et comprennent des espèces économiquement importantes (Oreochromis niloticus, Heterotis niloticus, Chrysichthys spp, etc.). Les valeurs de commercialisation montrent que la pêche représente une source non négligeable de numéraire pour ces villages.

Mots-clés : Côte d'lvoire, petits barrages, effort de pêche, production piscicole. 


\title{
CATCH-EFFORT AND FISH PRODUCTION IN SMALL DAMS IN THE NORTH OF THE IVORY COAST.
}

\begin{abstract}
The fishing-effort and the fish production have been studied in four agropastorai dams in the north of the Ivory Coast : Sambakaha (25 ha) and Gboyo (16 ha) in the region of Ferké, Kiémou (6 ha) and Korokara serpent (15 ha) in the region of North Korhogo. The studies have shown that the fishing is mostly done by Bozos from Mali. The fishermen density by dam, from 4 to 17 fishermen $\cdot \mathrm{km}^{-2}$, is well above FAO norms ( 2 fishermen $\cdot \mathrm{km}^{-2}$ ). The catch and the effort are highly variable from dam to dam. The fish production varies from 70 to $224 \mathrm{~kg} \cdot \mathrm{ha}^{-1} \cdot \mathrm{y}^{-1}$, which is in the same order of magnitude as the values registered in other small dams of the West African region. Yet, catch values are under FAO potential catches given for similar water bodies (between 100 and $750 \mathrm{~kg} \cdot \mathrm{ha}^{-1} \cdot \mathrm{y}^{1}$ ), indicating that there is a possibility of fish production increase. Total estimated catches in the small dams in the north of the Ivory Coast represent from 16 to $46 \%$ of total freshwater catches. Catches include economically important fishes (Oreochromis niloticus, Heterotis niloticus, Chrysichthys spp, etc.) and marketing values indicate that the fishing activity is an important source of currency for the villages.
\end{abstract}

Key-words : Ivory Coast, small dams, catch-effort, fish production.

\section{INTRODUCTION}

" Le poisson reste la principale source de protéines d'origine animale dans l'alimentation de la population ivoirienne : $42 \%$ dans les grandes agglomérations et presque $100 \%$ dans les campagnes (...) La consommation ivoirienne de poisson est de l'ordre de 250000 t/an. Cette consommation est déséquilibrée $(24 \mathrm{~g} / \mathrm{ind} / \mathrm{j}$ à $1 \mathrm{~g} / \mathrm{ind} / \mathrm{j}$ du sud au nord) et reste insuffisante par rapport à la norme internationale ( $30 \mathrm{~g} / \mathrm{ind} / \mathrm{j})$ " (ANONYME, 1989). Ce constat reste d'actualité. Les estimations pour l'année 1993 indiquent que la pêche continentale ivoirienne représenterait 13477 tonnes, soit 19,2\% des captures totales de poissons du pays, tous milieux confondus (ANONYME, 1993). La production locale des deux principaux départements (Korhogo, Ferké) du nord de la Côte d'Ivoire (1 072 tonnes) ne couvre pas la demande (16 800 tonnes) estimée pour la région. Elle ne représente que $7,9 \%$ de la production totale des pêches continentales de Côte d'Ivoire (ANONYME, 1993). Les petits barrages du nord de la Côte d'lvoire, dont le nombre avoisine 300 , y représentent la principale ressource en eau. Construits initialement pour l'irrigation des cultures et l'abreuvement du bétail, ces retenues jouent un rôle important dans l'approvisionnement des populations autochtones et riveraines en protéines d'origine animale. Elles représentent un potentiel halieutique exploité tout le long de l'année par de nombreux pêcheurs d'origines diverses (ANONYME, 1992 a). Mais il existe très peu d'informations sur le niveau d'exploitation des retenues hydroagropastorales, en particulier sur celles de petite ( $<10$ ha) et moyenne superficie (entre 10 et 50 ha) (NUGENT, 1997). En effet, dans la région nord de la Côte d'lvoire se pose un problème de fond pour le suivi des statistiques de pêche. Les pêcheurs refusent très souvent de fournir aux agents officiels chargés de l'encadrement et du contrôle des activités de pêche (Eaux et Forêts) leurs captures pour la pesée. Par ailleurs, ceux-ci manquent de moyens de fonctionnement et travaillent essentiellement au niveau des grandes retenues.

Un certain nombre de directives destinées à améliorer la planification et la gestion des plans d'eau ont été élaborées, notamment par la FAO (GIASSON et GAUDET, 1989 ; FAO, 1993, 1994 ; NUGENT, 1997). Toutes mettent l'accent sur la nécessité de mettre en 
place des études régionales prenant en compte les usages multiples des retenues et l'évaluation des risques et bénéfices associés à l'existence et à l'exploitation des barrages (voir en particulier VANDERPUYE, 1985). Avant la mise en place de l'étude à l'échelle de la région, il nous a paru important d'illustrer la situation présente dans des retenues de différentes tailles. Dans le cadre du programme IDESSAVCRDI (Institut des Savanes I Centre de Recherches pour le Développement International) sur les petits barrages du nord de la Côte d'Ivoire, quatre retenues ont été étudiées sur l'ensemble des vingt sélectionnées par I'IDESSA. L'effort de pêche exercé, la composition des captures et la production exploitée ont été analysés entre mai et décembre 1994. Les grandes retenues ont été écartées du dispositif pour des raisons logistiques et parce qu'elles sont moins représentatives de l'ensemble des retenues. Cette approche nous permet :

1) d'estimer si l'apport des petits barrages en termes de production piscicole est ou n'est pas significatif à l'échelle de la production annuelle des eaux douces du nord de la Côte d'Ivoire ;

2) d'estimer le niveau actuel d'exploitation des retenues en matière piscicole, en le comparant d'une part aux niveaux empiriques proposés par la FAO et d'autre part aux niveaux observés ailleurs ;

3) de déterminer, à partir de là, si l'effort de pêche actuellement en place est susceptible d'augmentation.

\section{MATÉRIEL ET MÉTHODES}

\section{Localisation et caractéristiques des retenues}

L'étude a été réalisée sur une petite ( $6 \mathrm{ha})$ et trois moyennes $(15,16$ et $25 \mathrm{ha}$ ) retenues situées en zone de savane, au nord de la Côte d'lvoire, dans les régions de Ferké et Korhogo (Figure 1) (ANONYME, 1992 b).

Ces quatre plans d'eau sont de type agropastoral. Ils ont été construits par la Société pour le Développement de la Production Animale (SODEPRA) sur les affluents du fleuve Bandama. Leur régime hydrologique en fait des barrages permanents et d'accès facile. Nous avons retenu pour chacun la donnée de surface et de profondeur la plus élevée parmi les données disponibles : surface maximale théorique (ANONYME, 1992 b) ou surface maximum observée (CORBIN, comm. pers.) ; profondeur maximum observée (CORBIN, comm. pers. ; YTE et al., 1997). Les marnages observés sont de $1 \mathrm{~m}$ environ en année normale.

La retenue de Sambakaha ( $25 \mathrm{ha}$, profondeur $3,0 \mathrm{~m}$ ) est située à $25 \mathrm{~km}$ de la ville de Ferké sur l'axe Ferké-Kong. Elle a été construite en 1984 sur la rivière Logahan, affluent secondaire du Bandama. Le barrage est situé à $500 \mathrm{~m}$ du village de Sambakaha.

La retenue de Gboyo ( $16 \mathrm{ha}$, profondeur $2,8 \mathrm{~m}$ ) est située dans la même zone géographique que celle de Sambakaha. Elle est située à environ $2 \mathrm{~km}$ du village d'Alamandjougoukaha. Cette retenue a été construite en 1984 sur la rivière Lagouho, affluent secondaire du Bandama.

La retenue de Korokara serpent (15 ha, profondeur $3,5 \mathrm{~m}$ ) est située à $63 \mathrm{~km}$ au nord de la ville de Korhogo, à proximité de la forêt classée du Badénou. Le barrage a été construit en 1976 sur la Kô qui est un affluent secondaire du Bandama. Le barrage est situé à $3 \mathrm{~km}$ au sud-est du village de Korokara. Un empoissonnement de cette retenue avec Oreochromis niloticus a été effectué en février 1985. 


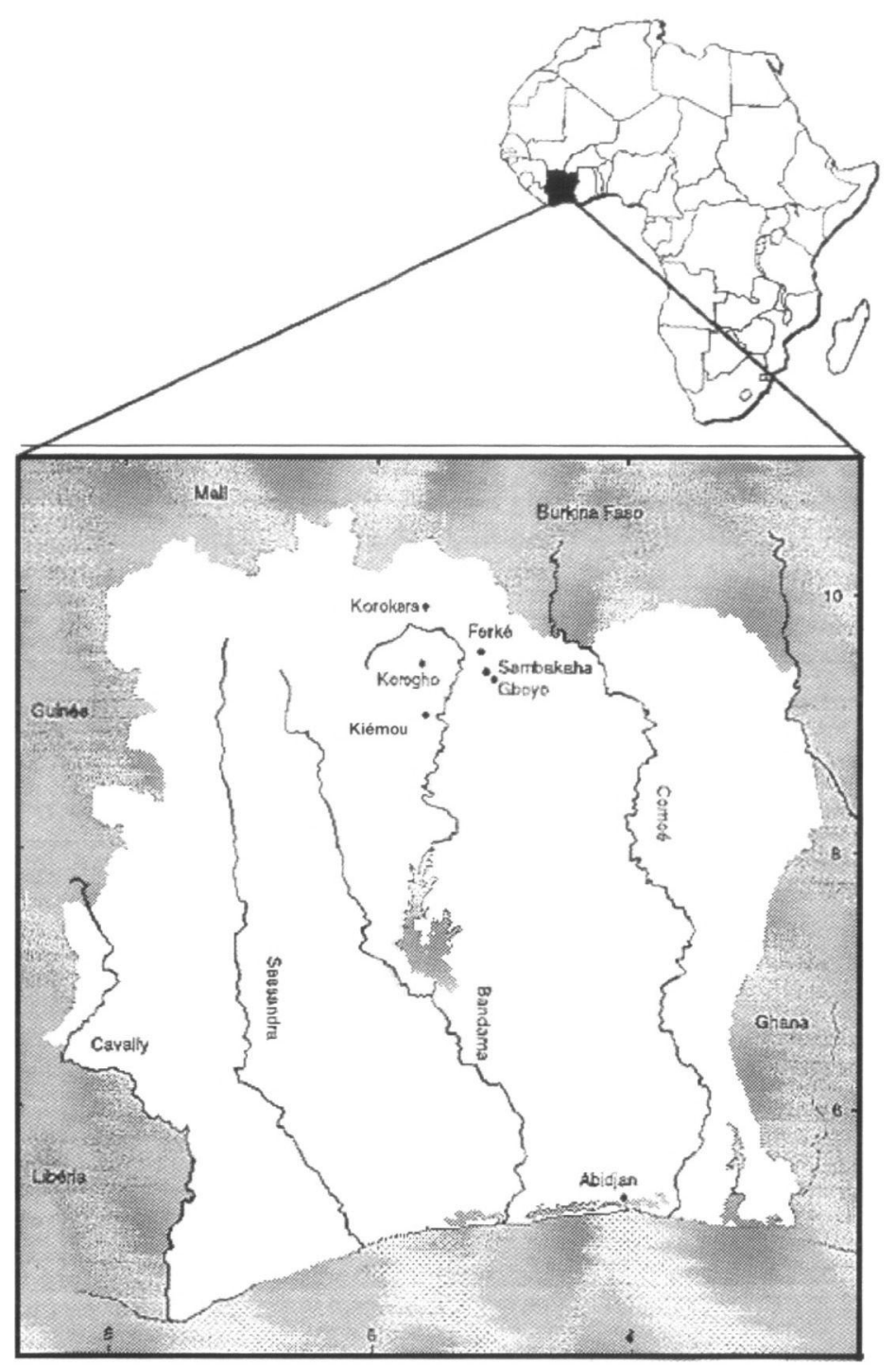

\section{Figure 1}

Carte de la Côte d'Ivoire indiquant la position des 4 barrages étudiés.

\section{Figure 1}

Map of the Ivory Coast with the position of the $\mathbf{4}$ study sites.

Le barrage de Kiémou aménagé ( $6 \mathrm{ha}$, profondeur $2,25 \mathrm{~m}$ ) est situé à $55 \mathrm{~km}$ au sud de Korhogo sur l'axe Napié-Sohakaha. Il a été construit en 1983 sur la rivière Foro, petit affluent du Solomougou, lui-même affluent du Bandama. Cette retenue a fait l'objet d'empoissonnements répétés avec Oreochromis niloticus en octobre, novembre et décembre 1984, et janvier et février 1985. Le barrage est situé à $2,5 \mathrm{~km}$ du village de Kiémou.

D'autres empoissonnements sur lesquels il n'y a pas de données disponibles ont été réalisés en utilisant $O$. niloticus et Heterotis niloticus. Ces deux espèces sont présentes dans les quatre retenues. 


\section{MÉTHODE D'ÉTUDE}

Les enquêtes de pêche ont été conduites pendant 8 mois, de mai à décembre 1994, sur les 4 retenues. Le contrôle de la pêche a consisté à faire relever chaque jour par un enquêteur local l'ensemble des captures des pêcheurs de chaque retenue. Les enquêteurs sélectionnés ne sont pas des professionnels, ils ont pour activité principale l'agriculture. Sur le barrage de Korokara serpent, cette activité a contrarié les enquêtes et n'a pas permis un suivi quotidien des pêches. Dans ce barrage, seuls 2 à 3 jours par semaine ont fait effectivement l'objet d'enquêtes. La limitation de l'enquête dans le temps (mai à décembre) est également liée à la disponibilité des enquêteurs. Cette limitation ne devrait pas invalider nos conclusions, le but de l'étude n'étant pas une analyse fine des pêcheries mais bien d'avoir les ordres de grandeur des volumes mis en jeu tant en poids des captures qu'en valeur monétaire.

Le recensement des pêcheurs, le nombre d'unités de pêche par retenue et l'inventaire des engins utilisés ont été réalisés au début de l'étude, en mai (fin de l'étiage, début des pluies) puis en septembre (hautes eaux). Le type et les dimensions des engins utilisés ont été notés ainsi que les espèces recherchées. Entre ces périodes, les mouvements des pêcheurs et des engins de pêche ont été suivis. Le résultat de la pêche a été recensé comme suit : poids total par jour de pêche, poids total par engin, poids total par espèce.

\section{RÉSULTATS}

\section{Catégories de pêcheurs}

Les populations autochtones riveraines, les Sénoufos et Dioulas (Korokara), ne pratiquent pas de manière générale la pêche. Un seul pêcheur Sénoufo a été enregistré sur la retenue de Sambakaha. Ce cas est unique sur l'ensemble des retenues étudiées par l'IDESSA. Les pêcheurs sont dans leur grande majorité des étrangers, pour la plupart maliens, connus sous le nom de "Bozos " bien que n'appartenant pas tous à cette ethnie. Trois catégories de pêcheurs ont été identifiées sur les barrages étudiés (Tableau I) en fonction du temps consacré à l'activité de pêche : (1) pêcheurs professionnels exerçant à plein temps et dont les revenus financiers sont exclusivement issus de la vente des poissons ; (2) pêcheurs semi-professionnels exerçant la pêche à temps partiel mais dont la pêche est néanmoins l'activité principale, et (3) des pêcheurs occasionnels.

\section{Régime d'exploitation}

L'exploitation halieutique des 4 retenues étudiées se caractérise par des rythmes irréguliers (Figure 2). De nombreux conflits apparus entre comités de gestion des barrages et pêcheurs ont considérablement perturbé l'activité de pêche. A Korokara serpent, cela a valu un arrêt de la pêche durant le mois de juin, suivi d'un arrêt total d'août à décembre. $A$ Sambakaha, la mésentente entre le comité de gestion et les pêcheurs a conduit au boycott de la retenue du village par tous les pêcheurs Bozos de la région de Ferké durant la période d'étude. De ce fait, il n'y a pas eu de pêche de mai à juin. A partir de juillet 94, le comité de gestion a sollicité un pêcheur autochtone (Sénoufo) pour exploiter la retenue. Sur la retenue de Kiémou, les conflits sont d'ordre relationnel avec seulement de brèves périodes d'arrêt. A Gboyo, aucun conflit n'a été noté pendant la période d'étude. Les quelques autres arrêts de pêche sont dûs à des facteurs comme la maladie, les voyages des pêcheurs, l'indisponibilité temporaire des engins de pêche abîmés, etc. 
$30 \mathrm{~mm}$ de vide de maille. Son utilisation est épisodique et se fait en accord et parfois à la demande du comité de gestion de la retenue. La senne peut être également utilisée en dehors de son barrage d'origine (à Gboyo en juillet 94 ). Son utilisation est limitée et rendue délicate par l'encombrement des lacs non déboisés lors de la mise en eau.

\section{Production exploitée}

\section{Taxons capturés}

Les poissons capturés appartiennent à 11 familles (Tableau III) et plus de 17 espèces. Certaines déterminations, réalisées par les enquêteurs, ont été maintenues au niveau du genre - plus rarement de la famille - par mesure de prudence. Ce niveau de précision est suffisant pour le présent travail qui n'aborde pas les aspects biologiques mais la production globale des retenues et son exploitation. Cinq taxons sont ubiquistes (Sarotherodon galilaeus, Tilapia zillii, Clarias spp, Oreochromis niloticus, Heterotis niloticus), les deux dernières espèces étant des espèces introduites. Le barrage de Sambakaha a la richesse spécifique la plus élevée.

\section{Tableau III}

Familles et taxons dans les captures des pêcheurs des barrages étudiés. + : espèce présente dans les captures.

Entre parenthèses : rang et poids $(\mathrm{kg})$ dans les captures.

\section{Table III}

Families and fish taxa in the catches in each studied dam.

+ : species present in the catches.

In brackets : rank and weight $(\mathbf{k g})$ in the catches.

\begin{tabular}{|c|c|c|c|c|c|}
\hline Famille & Taxon & Gboyo & Kiémou & Korokara s. & Sambakaha \\
\hline Anabantidae & Ctenopoma kingsleyae (Günther 1896) & & & + & \\
\hline \multirow{5}{*}{ Cichlidae } & Chromidotilapia guntheri (Sauvage 1882) & & & + & \\
\hline & Hemichromis tasciatus (Peters 1856) & (4 100) & $+(2.315)$ & $\stackrel{+}{+}$ & $+(7-130)$ \\
\hline & Oreochromis niloticus & $+14-1001$ & & & $+(4-230)$ \\
\hline & Sarotherodon galilaeus (Linné 1758) & $+(5-100)$ & + & + & $+(6-160)$ \\
\hline & Tilapia zillii (Gervais 1853) & $+(2-130)$ & $+(1-530)$ & $+(5-40)$ & $+(1-645)$ \\
\hline \multirow[t]{2}{*}{ Clariidae } & Clarias spp & $+(1-190)$ & + & $+(4-40)$ & $+(5-175)$ \\
\hline & Heterobranchus spp & & & + & + \\
\hline Claroteidae & Chrysichthys spp & & & $+(2-60)$ & $+(2-540)$ \\
\hline Cyprinidae & Barbus spp & & + & & + \\
\hline Hepsetidae & Hepsetus odoe (Bloch 1794) & + & & & + \\
\hline Malapteruridae & Malapterurus electricus (Gmelin 1789) & & & & + \\
\hline Mochokidae & Synodontis spp & $+(3-115)$ & & + & + \\
\hline Mormyridae & Marcusenius spp & $+(6-80)$ & & + & + \\
\hline & Petrocephalus bovei (Valenciennes 1846) & + & & + & + \\
\hline Osteoglossid & Heterotis niloticus (Müller 1843) & + & + & $+(1-120)$ & $+(3-250)$ \\
\hline Schilbeidae & Schilbe spp & + & & + & + \\
\hline 11 familles & 17 taxons & 11 & 7 & 14 & 15 \\
\hline \multicolumn{2}{|c|}{ Richesse spécifique totale } & 24 & 10 & 24 & 26 \\
\hline
\end{tabular}


Du point de vue pondéral, les taxons dominants sont, par ordre d'importance : T. zillii, O. niloticus, Chrysichthys spp, Clarias spp, $\mathrm{H}$. niloticus et $\mathrm{S}$. galilaeus (Tableau III). Les autres espèces interviennent dans les captures en quantité moindre.

\section{Production par type d'engin}

Les trois principaux engins de pêche utilisés sur les retenues pendant la durée de l'étude sont : l'épervier, les filets maillants et les mitraillettes (grandes palangres de fond) (Figure 3). De façon générale, les captures mensuelles totales à l'épervier régressent avec la montée des eaux sur les barrages étudiés. Les captures aux filets maillants croissent quant à elles avec la montée des eaux : partant d'une production pratiquement nulle en fin d'étiage, les captures mensuelles totales sont plus importantes entre août et décembre. Le pic des captures totales mensuelles aux mitraillettes est atteint en hautes eaux. Avec la montée des eaux, les zones de rivages sont inondées, ce qui favorise l'usage de cette technique de pêche. Cette tendance est aussi observée avec les nasses dont la production croît avec la montée des eaux. La senne a été utilisée une seule fois pour les pêches en juillet sur les retenues de Gboyo et Sambakaha avec des captures respectives de 74 et $18 \mathrm{~kg}$. Les espèces inventoriées dans les quatre retenues sont pour la plupart représentées dans les prises des différents engins de pêche. Les filets maillants et la senne en particulier capturent en général toutes les espèces. Les autres engins sont plus sélectifs. Quelle que soit la retenue, l'épervier capture principalement $O$. niloticus, $T$. zillii et $S$. galilaeus. Les Clarias spp, Chrysichthys spp et $H$. niloticus apparaissent en quantité moindre. Les mitraillettes sont très sélectives pour les Clariidae et autres Siluriformes. Les nasses exercent une sélectivité importante sur les Cichlidae et les Siluriformes.

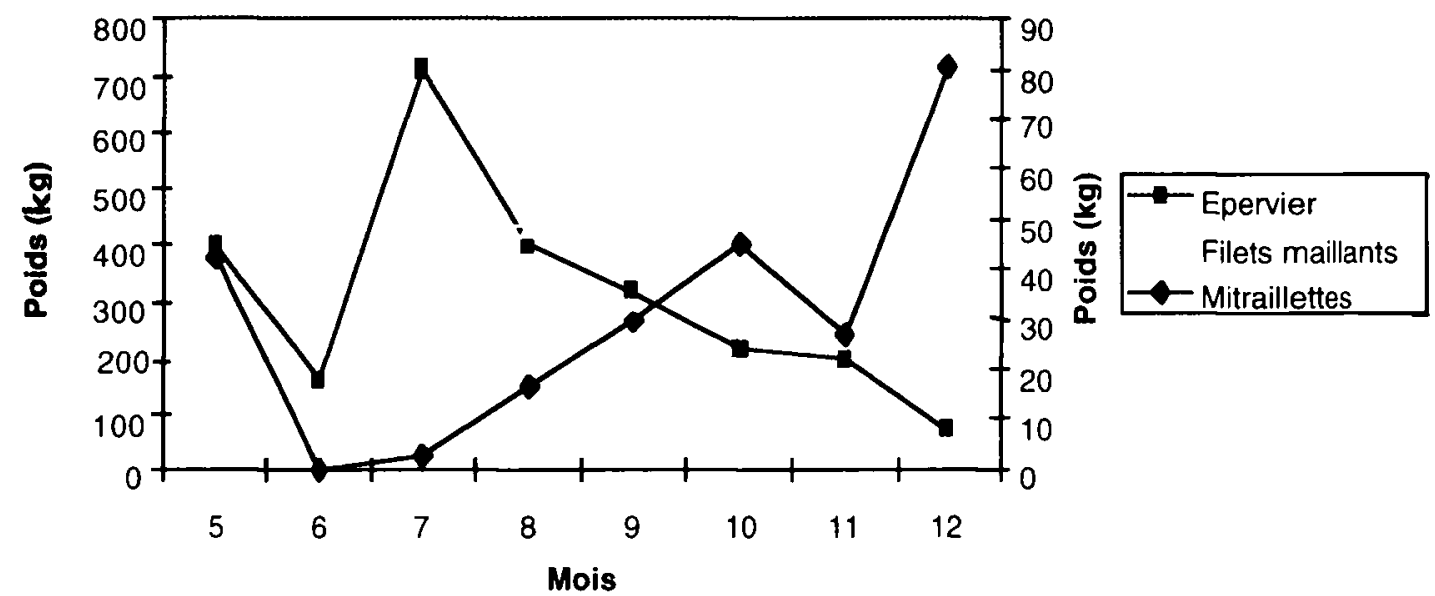

\section{Figure 3}

Poids des captures mensuelles (en $\mathrm{kg}$, pour toutes les espèces dans les 4 barrages) par engin de pêche. Echelle de gauche : épervier. Echelle de droite : filets maillants et mitraillettes.

\section{Figure 3}

Weight of the monthly catches (in $\mathrm{kg}$, for all species in all 4 dams) for each fishing gear. Left scale : longlines. Right scale : gillnets and castnets. 
production reste cependant faible par rapport aux captures potentielles évoquées par la FAO pour les petits barrages, entre 10 et $75 \mathrm{t} / \mathrm{km}^{2} / \mathrm{an}$ (PALM, 1989). Même si cette valeur supérieure est excessive, une valeur intermédiaire entre 30 et $40 \mathrm{t} / \mathrm{km}^{2} / a n$ indique qu'il existe une certaine marge pour augmenter la productivité des petites retenues du nord de la Côte d'Ivoire par comparaison avec les captures atteintes ailleurs en milieu tropical (Tableau IV).

Les modalités de gestion actuelles sont très diverses et mal appréhendées sous l'angle socio-ethnologique (KOFFI et al. in ANONYME, 1992 a). La mauvaise définition légale de la propriété est souvent à l'origine de multiples conflits entre pêcheurs et comités de gestion, parfois exacerbés par la nationalité étrangère des pêcheurs (malienne pour la plupart). La formation de pêcheurs villageois locaux et une meilleure définition des droits et obligations de chacun sont de nature à améliorer les relations et donc la gestion, piscicole entre autres, des retenues. II importe cependant de tenir compte du fait que le niveau d'effort de pêche actuel en termes de nombre de pêcheurs par unité de surface (Tableau I) est déjà élevé par comparaison avec les normes FAO (2 à 3 pêcheurs $/ \mathrm{km}^{2}$ ) (HENDERSON et WELCOMME, 1974 ; KNAAP, 1994). Les valeurs observées ici (4 à 17 pêcheurs $/ \mathrm{km}^{2}$ ) sont voisines de celles observées dans les petites retenues au Burkina Faso (3 à 11 pêcheurs $/ \mathrm{km}^{2}$ ) (BAIJOT et al., 1994) et il est donc impossible de jouer sur ce paramètre pour augmenter la production des retenues. L'efficacité de la pêche est certainement un facteur sur lequel il est possible de jouer. Les engins que nous avons observés en cours d'utilisation sont en effet en très mauvais état. Cependant, dans les cas où une surpopulation de pêcheurs est observée, toute intervention conduisant à une meilleure efficacité des pêcheurs ne devra être entreprise qu'avec des mesures d'accompagnement adéquates. Nous n'avons actuellement pas de connaissances sur la productivité des retenues et ce point est en cours d'étude. II existe très peu d'articles scientifiques analysant les pêcheries des petits barrages africains, le plus grand nombre provenant des colloques de la FAO (GIASSON et GAUDET, 1989). Au-delà des aspects sociologiques ou réglementaires (NUGENT, 1997) qui dépassent le cadre de cette étude, les facteurs susceptibles d'inflexions permettant une exploitation plus rationnelle des retenues sont : la formation des intervenants (pêcheurs et membres des comités de gestion), la qualité des engins et des actions sur le plan biologique (bio-manipulations, manipulations de I'habitat, empoissonnements, contrôle de la qualité des eaux). Ces derniers points demandent cependant une bien meilleure connaissance du fonctionnement biologique des retenues afin de ne pas constituer, comme trop souvent par le passé, des interventions coûteuses et sans lendemain. Le tableau contrasté décrit ici indique en effet que tout plan de gestion devra être établi par retenue en fonction de sa spécificité locale, biologique et culturelle.

\section{REMERCIEMENTS}

Les auteurs remercient I. DEMBÉLÉ, Technicien supérieur à I'IDESSA, pour son aide sur le terrain.

\section{BIBLIOGRAPHIE}

ANDERSON A., 1989. The development and management of fisheries in small water bodies. In GIASSON M., GAUDET J.L. (eds), Summary of proceedings and selected papers. Symposium on the development and management of fisheries in small water bodies. Accra, Ghana, 7-8 December 1987. FAO Fish. Rep./Rap. FAO Pêches, 425, 15-19. 
ANONYME, 1989. Ministère des Eaux et Forêts. La pisciculture et les pêches continentales en Côte d'lvoire. In GIASSON M., GAUDET J.L. (eds), Summary of proceedings and selected papers. Symposium on the development and management of fisheries in small water bodies. Accra, Ghana, 7-8 December 1987. FAO Fish. Rep./Rap. FAO Pêches, 425, 74-78.

ANONYME, 1992 a. Valorisation du potentiel piscicole des barrages hydro-agropastoraux du nord de la Côte d'Ivoire. IDESSA/CRDI. Rapport préliminaire IDESSAR, Bouaké, Côte d'Ivoire, multigr., $181 \mathrm{p}$.

ANONYME, 1992 b. Inventaire des barrages hydro-agricoles et à autres vocations existant en Côte d'Ivoire. Direction et Contrôle des Grands Travaux (DCGTx). Rapport, Abidjan, Côte d'Ivoire, multigr., $57 \mathrm{p}+$ annexes.

ANONYME, 1993. Annuaire des statistiques des pêches et de l'aquaculture maritimes et lagunaires. Ministère de l'Agriculture et des Ressources Animales, Abidjan, Côte d'Ivoire, multigr., $56 \mathrm{p}$.

BAIJOT E., MOREAU J., BOUDA S., 1994. Aspects hydrobiologiques et piscicoles des retenues d'eau en zone soudano-sahélienne. Centre Technique de Coopération Agricole et Rurale (CTA), Commission des Communautés Européennes (CCE), Bruxelles, $250 \mathrm{p}$.

BERNACSEK G.M., 1984. Dam design and operation to optimize fish production in impounded river basins. Based on a review of the ecological effects of large dams in Africa. CIFA Tech. Pap., FAO, Rome, Italy, 11, $98 \mathrm{p}$.

CLAY C.H., 1984. Nouveaux réservoirs africains 1980-2000. CIFA Occ. Pap., FAO, Rome, Italy, 11, $23 \mathrm{p}$.

FAO, Committee for Inland Fisheries of Atrica, 1993. Report of the fifth session of the subcommittee for the protection and development of the fisheries in the Sahelian zone. FAO Fish. Rep., 504, $31 \mathrm{p}$.

FAO, Committee for Inland Fisheries of Africa, 1994. Report of the working party on pollution and fisheries. Accra, Ghana, 18-22 October 1993. FAO Fish. Rep., 502, $45 \mathrm{p}$.

GERLOTTO F., STEQUERT B., 1978. La pêche maritime artisanale en Afrique de l'Ouest. Caractéristiques générales. La Pêche Maritime, 278, 278-285.

GIASSON M., GAUDET J.L. (eds), 1989. Summary of proceedings and selected papers. Symposium on the development and management of fisheries in small water bodies. Accra, Ghana, 7-8 December 1987. FAO Fish. Rep., 425, 190 p.

HENDERSON H.F., WELCOMME R.L., 1974. The relationship of yeld to morpho-edaphic index and number of fishermen in African inland fisheries. CIFA Occ. Pap. FAO, Rome, Italy, 1, $19 \mathrm{p}$.

JACKSON P.N.B., BRUTON M.N., 1988. Peuplements ichtyologiques dans les lacs de barrages. In LÉVÊQUE C., SSENTONGO G.W. (eds), Biologie et écologie des poissons d'eau douce africains, 325-350, Trav. Inst. Fran. Rech. Dévl., 216, Paris.

KNAAP Van der M., 1994. Status of fish stocks and fisheries of thirteen medium-sized African reservoirs. CIFA Tech. Pap., 26, $107 \mathrm{p}$.

KOFFI C., BOBLAI B., SORO FIASSORO L., 1992. Aspects socio-économiques des pêches et de la commercialisation des ressources des plans d'eau hydroagropastoraux du nord de la Côte d'lvoire. In ANONYME, Valorisation du potentiel piscicole des barrages hydro-agropastoraux du nord de la Côte d'Ivoire, 144-181, IDESSA/CRDI. Rapport préliminaire IDESSA, Bouaké, Côte d'Ivoire, multigr., 181 p.

MOREAU J., DE SILVA S.S., 1991. Predictive fish yield models for lakes and reservoirs of the Philippines, Sri Lanka and Thailand. FAO Fish. Tech. Rep., 319, 42 p. 
NUGENT C.G., 1997. Assistance en matière de législation portant sur les pêches continentales. Rapport sur l'aménagement des pêches continentales. Côte d'Ivoire. Programme de Coopération Technique, FAO., Rome, Italie, TCP/IVC/4553, 86 p.

PALM R., 1989. Management of community small water bodies for fish production in Africa. In GIASSON M., GAUDET J.L. (eds), Summary of proceedings and selected papers. Symposium on the development and management of fisheries in small water bodies. Accra, Ghana, 7-8 december 1987. FAO Fish. Rep./Rap. FAO Pêches, 425, 139-169.

VANDERPUYE C.J., 1985. Evaluation guidelines for rational planning and management of tropical and subtropical inland fisheries under constraints from other uses of land and water resources. Africa. FAO Fish. Circ., 789, $44 \mathrm{p}$.

YTE W.A., KOUASSI N.C., AYEKOUE P., SORO S., 1997. Peuplement zooplanctonique de quelques retenues agropastorales du nord de la Côte d'lvoire : les rotifères. Agron. Afr., 9 (2), 125-134. 